KEY WORDS

1. Satellite navigation. 2. Interference. 3. Reliability.

\title{
Drs Ward and Johannessen comment:
}

It is not possible from Captain Gylden's contribution to fully explain the phenomenon. It is, however, important to note his experience and to encourage other practical navigators to report similar events. It is essential to have the best possible picture of circumstances when GPS suffers problems, before it is decided to close down alternative systems, which might not be affected in the same way.

\section{Editor's note}

Reports of GPS outages or other operational problems experienced by practical navigators would, as suggested by Drs Ward and Johannessen, be welcomed by the Institute. To be of maximum value for subsequent analysis, reports should give a complete as possible description of the circumstances. The following information would always be useful:

(1) Date and time of day (GMT).

(2) The observer's position.

(3) Make, model and other details of the receiver.

(4) The antenna used, its position and a note of any obstructions in the vicinity which might have masked part of the sky.

(5) The power supply.

(6) The weather conditions.

(7) Whether the receiver was using GPS or DGPS.

(8) A description of the abnormality being reported. For example if the display showed wrong information or no information. Was there a message saying 'power down and re-initialize?' How long the condition lasted and whether it recovered by itself, etc.

(9) Whether any other electrical equipment which might have caused interference to the GPS was in use at the time. For example, was HF, MF of VHF equipment being operated and, if so, on what frequency?

(10) Any other information which might be relevant. 